\title{
A Web Based Course on Designing High Density Interconnect PCBs for Manufacturability
}

\author{
G.Ananda Rao and N.J.Rao \\ Centre for Electronics Design and Technology, Indian Institute of Science, Bangalore 560012, India.
}

\begin{abstract}
The design of high density interconnects (HDI)

PCBs is an important step in design and development of present day electronic products. This design is becoming a complex task with the availability of a large number of process technologies. Each technology offers its own cost (yield)/volume/performance optimisation. The designer should be able to do the package design in the context of a selected manufacturing technology, and communicate effectively with the fabricator. This process may be termed as "design for manufacturability" (DFM). While DFM issues are not new to an electronic/PCB designer, these acquire new importance in the context of proliferation of manufacturing processes and increasing performance requirements. In addition, these issues are in a constant state of flux, as process changes and new processes are being introduced on a continuing basis. Consequently, there is a need for the designer to continuously keep track of process improvements, and take advantage of them to make his product more competitive. This paper presents the structure of a course on "design of HDI PCBs for manufacturability". While it is designed as a second level course on electronic packaging, that can be offered at graduate level. As a larger number of practising engineers are likely to be interested in such a course, it is proposed to make it web based.
\end{abstract}

\section{Introduction}

Present day electronic products are characterised by miniaturisation, high performance, portability, continuously decreasing costs, and short lead times to market. Most of the products require the use of high-density high-performance PCBs. The drive to increase the silicon efficiency is leading to a proliferation of chip level packages, different ways of incorporating passive components, and a multiplicity of manufacturing (substrate and assembly) processes. The final performance and cost effectiveness of the final product is significantly influenced by packaging design and the manufacturing technology used. Functional designer has to have an appreciation of these influences. Board level packaging designer needs to understand how the packaging design would influence the performance and reliability of the product, and the possibilities and limitations of different manufacturing processes. The short lead times demand that the packaging design be done while addressing the manufacturability issues. This approach may be called as "design for manufacturability" (DFM).

Design of high density interconnect PCB for manufacturability has to take several factors into consideration simultaneously. These include electrical performance, thermal behaviour, ease of testing, reliability, suitability to the selected manufacturing process, and documentation. Therefore the packaging designer has to have adequate understanding of the materials used and the manufacturing processes, and their influence on the reliability, besides his proficiency in performing the package design using the state-of-the-art CAE/CAD tools. This paper identifies many factors that influence the design of a high density interconnect PCB, and makes a case for a course that uses the DFM framework to train packaging designers of such PCBs. As majority of the designers needing this input are likely to be practising engineers, and the design process requires access to a wide range of information, a web based learning methodology is proposed.

\section{High Density Interconnect Boards}

High density interconnect (HDI) PCBs are characterised by high frequency operation, interconnect line widths between 50 and 100 microns, micro vias ( 50 microns) which can be through, buried or blind, multilayer substrate, with possibly passives integrated at the substrate level, higher heat dissipation densities, and a wide variety of chip level packages. HDI PCBs may, in near future, have to handle optical interconnects as well.

Emergence of high density interconnections at the board level is the consequence of the changes occurring at the chip package level, and new approaches such as multichip modules (MCMs), chip scale packages (CSPs), flipchip-on-laminate (FCOL), chip-on-board (COB), and direct chip attach (DCA). In addition to these approaches the package pin count has gone up from DIP-64 to 500 in the case of peripheral quad packages. The peripheral packages, in turn, are slowly yielding their place to area array packages. The drive for such a shift is the design and assembly difficulties associated with high pin count peripheral packages. In contrast, the area array packages provide greater functionality with much coarser pitches but bring with them other difficulties such as addition of a large number of blind via interconnections. For example, a high density board using chips packaged as "ball grid arrays" to take advantage of the small ball pitch will require extensive use of blind vias.

\section{Technology Options}

At present, more than 12 substrate technologies are available for manufacturing high density interconnect boards that can accommodate all types of packages, including BGAs. The design issues vary slightly from process to process, so that the possibility of designing to one technology, and having it built by another would not be appropriate. Unlike earlier times, there can not be anything like a universal design. The designer needs to understand the advantages of a particular manufacturing environment and generate a design that represents the most cost-effective solution for a given end application and a manufacturing 
environment. Substrate technologies can be predominantly classified according the manner in which micro vias are created. The photo-defined micro-via is the first and the simplest structure. The layers are added sequentially which provide immense opportunity for blind and buried vias, which are so essential for HDI design. When the layer count is beyond 6 layers, the photo-defined technology is likely to become uneconomical due to sequential building of layers. The design for photo-micro via should also consider the coplanarity element, and the limitation of epoxy as dielectric. The designer should be sensitive to these issues, in addition to via size process limitation, the inter-boundary adhesion failures, and limitations of introducing thermal planes. The laser defined micro-via technology can be of sequential or conventional multi layer construction. There are plenty of variations, depending on using either copper deposition or conducting adhesive for via connections. In fact Any Layer Inner Via Hole [ALIVH] is laser based with conducting adhesive replacing copper plating for via hole connection. Higher layer count is a good possibility, and the general economics indicates suitability for large volume products. The plasma-based technology is an excellent alternative for making via holes, but the cycle time and panel size are the limitations. Typical cycle time is about 20 minutes, and the plasma chamber has to be large enough to accommodate the selected panel size. The advantage of using plasma is clean hole wall definition with assured copper adhesion for greater reliability. There are variants like 3-M technology, Tessera Technology, Sheldhal Technology which use chemical milling, interlayer interposer, or Z-axis anisotropic adhesive for establishing micro-vias. Each technology offers a different real estate gains and cost advantages. With respect to a given technology, the gains in real estate area and cost are dependent on the design, as exemplified in the Table 1.

The circuit board assembly processes (component handling and soldering) are very much dependent on the choice of component (ICs and passives) packaging. A large variety of equipment is used for the assembly of circuit boards. Different automated assembly equipment requires different clearances, depending on the jigs used, for placement of components. The parameters associated with these assembly processes are generally equipment specific. It would be difficult to evolve any general principles with regard to design rules in relation to assembly equipment. The designer, by and large, has to evolve his own rules in the context of the $\mathrm{CAD}$ package he is using, and the equipment used for assembling the boards.

Table 1: Cost/Board-areas associated with different process technologies

\begin{tabular}{|l|c|c|c|c|}
\hline \multirow{2}{*}{ Technology } & \multicolumn{2}{|c|}{ DESIGN 1 } & \multicolumn{2}{c|}{ REDISIGN } \\
\cline { 2 - 5 } & $\begin{array}{c}\text { Board } \\
\text { Area }\end{array}$ & Cost & $\begin{array}{l}\text { Board } \\
\text { Area }\end{array}$ & Cost \\
\hline Conventional MLB & 100 & 100 & 100 & 100 \\
\hline Photo-via & 82 & 72 & 77 & 69 \\
\hline Laser-via & 71 & 60 & 68 & 58 \\
\hline ALIVH & 66 & 46 & 62 & 44 \\
\hline Plasma-via & 68 & 54 & 68 & 54 \\
\hline
\end{tabular}

\section{Design of HDI PCBs}

While all the design steps are interrelated, and have to be resolved in the context of DFM framework, the design can be considered to be consisting of the following four steps

- Electrical design

- Thermal design

- Design of testability

- Documentation

In the context of the decisions taken by the product development team with regard to

- component packaging (chip package, SMD, IMC or mixed)

- type of board (SS rigid, DS rigid, MLB rigid, flex, rigid-flex, 3-D etc.)

- substrate technology

- assembly technology

- board finish (HAL, OSP, Ni/Au etc.)

- reliability specifications

Some aspects of the four design tasks, seen from the manufacturing point of view, are presented in the following.

\section{Electrical Design}

Electrical graduate engineer is generally more sensitive to this requirement, which is best addressed by using appropriate software package. The chief issue addressed is signal integrity, which include parameters such as cross talk, reflections, and propagation delay. These issues are sensitive to design, materials, and manufacturing abilities in a sense that dielectric property, dielectric separation, conductor side wall definition, and planarity of the board will be contributing factors. Simulation $\mathrm{CAD}$ packages are helpful, provided the designer is able retro-fit latitudes of material properties and process abilities.

\section{Thermal Design}

The thermal requirement is a new dimension to electrical engineers learning. In HDI design in particular, thermal management becomes very significant. Small size packages means higher power densities ( $4-10$ watts/sq.cm.). There are two levels of cooling which the board designer need to consider. They are cooling of the component from the top using external heat sinks, and the other is the cooling of the component from the bottom by using speciality substrate material for board construction. While the $\mathrm{CAD}$ packages will assist the designer in mapping the thermal profile of the board for a given design, the designer should be able to select substrate materials for the relevant layers, and the heat dissipation methodology.

\section{Design for Testability}

Testing HDI boards has remained as a challenge. The board unless tested cannot be qualified for manufacturing. There are two levels of testing; the bareboard, and the populated board. The designer has to consider both these issues while working on the component layout itself. Providing test nets and test-nodes without using much real estate area could be a tricky job, and again the CAD platform is not likely to assist him. Therefore, knowledge of 
methods of testing becomes a prerequisite for the designer. The design for bed-of-nails testing, and flying-probe testing are completely different.

\section{Documentation}

Documentation is the link between the designer and the manufacturer. Quite often the design failure is because of inadequate documentation. The purpose of documentation is to provide sufficient information to the manufacturer to enable him to build the board without calling the customer for discussions. For every item required, but not included in the document will result in dissatisfaction to both. CAD/CAM package or EDA tools can care of this; but the problem here is the lack of consensus among the industry standards in areas related to data exchange necessary for machine to machine dialogue. New information is required as new technologies drive the change. The designer should constantly upgrade his knowledge of such specifications and standards related to materials, board performance, quality assessment, component mounting, testing, and workmanship.

Implications of some designs are listed in the following, to indicate the nature of DFM issues in packaging design.

- Component selection decides assembly technology, board cleaning technique, board density, reliability and testing.

- Component layout decides tooling for testing, soldering method and cooling methods.

- Substrate selection decides the process technology.

- Decision to use a thermal plane decides the structure of the board.

- Via size decides the drilling methodology.

- Number of drill sizes influences the productivity.

- Via locations decide reliability, soldering yields and PTH reliability.

- Type of print decides the soldering technology.

- Board finish influences the planarity of the board.

Therefore, the closer the designer understands the manufacturing principles, the better off he/she is when it comes to meeting the projections and market windows into which their product is being aimed and released. One has to strike a delicate balance between use of a $\mathrm{CAD}$ tool, and his/her own understanding of the manufacturing constraints.

Whereas $\mathrm{CAD}$ packages are available as self-learning exercises, the DFM issues will not be so obvious to the designer. Hence, there is need for course training a designer for designing HDI PCBs in the framework of DFM.

The cost reduction of the board cannot be controlled by the manufacturers alone. The board designer plays a very significant part in cost reduction. The traditional serial approach to design where the design proceeds from the logic circuit to physical designer [CAD layout] to manufacturing floor, and finally to test engineer is not appropriate because each engineer is working by himself/herself, and making decisions independently when evaluating and selecting alternatives. An ideal path would be a parallel or a concurrent design approach, where logic designer, CAD layout designer, manufacturing, testing and process engineers, and purchasing as well as marketing representatives interact with each other on a regular basis to review the design, and discuss the alternatives to meet thermal, electrical, real estate, cost and time-to-market requirements. The need for such a course exists, as it is the early decisions taken by electrical circuit designer, which will ultimately limit all other decisions down the chain. In the normal course, the electrical designer engineer is more comfortable to work in an atmosphere where the others involved in the product development should provide him a set of design-rules or default values for establishing interconnections. Such rules when provided by the manufacturing/test. engineers will have so much of elbowroom that any design based on this alone will rarely make the overall design optimum. Therefore, any attempt on optimisation, which is very essential to remain costcompetitive, is possible only when the electrical designer picks the ability to judicially convert the rules into guidelines for optimisation. The purpose of the course is therefore, to enable the electrical design engineer to design his circuit for optimal cost/performance effective manufacturing.

In web-education it is easier to bring the manufacturing atmosphere on the $\mathrm{PC}$ screen through video clippings.

\section{Structure of the course}

The target audience for the course is essentially graduate engineers. These engineers generally have only limited knowledge of materials, processes, assembly, and testing required for producing a printed circuit board. Therefore, there is need to provide more support on these issues, starting almost from fundamental levels. The course should also emphasise integration of the derived knowledge for the purpose of manufacturing. It is believed that as the designer experiences the design more and more, he would feel further interested in exploring further the material and manufacturing issues. Therefore, it is desirable to divide the depth of coverage in each topic into at least two levels, leaving scope for the student to choose between either just enough information or get into more details. The course is divided into a number of modules, each module is treated at two levels of coverage.

\section{Name of the Module \\ Hours Hours}

1. Materials and packages -

high performance needs

2. Overview of manufacturing processes

for HDI PCBs with micro-vias

3. Assembly of HDI PCBs

4. Thermal behaviour of HDI-PCBs

5. Electrical behaviour of HDI-PCBs

6. Testing of HDI-PCBs

7. Integrated approach for HDI-PCBs

8. Design documentation

Level 1 Level 2

Total lectures
44

22

22

22

$2 \quad-$

$2-$


The Laboratory sessions will include:

1. CAD for HDI-PCBs 5

2. Testing of HDI-PCBs 2

3. Design decision analysis 2

Total number of exercises

The lectures will essentially introduce the designer to the state-of- the-art of core manufacturing issues, with corresponding laboratory, culminating in a critical analysis of the design decision taken. The syllabus of each module is outlined below:

Module 1: High performance materials [2 $+2 \mathrm{hrs}]$ Role of substrates in PCBs. Types of substrates. Properties of substrates of interest to designer. Limitation of conventional substrates. Description of high performance requirement. Application of design parameters.

Module 2: Manufacturing processes for HDIPCBs[6+2 hrs] Outline of PCB manufacturing. Routes for manufacturing. Subtractive, additive routes - advantages and limitations. Understanding of high-density interconnection. Micro-via technology. Via types and ease of manufacture. Limitations at every process step. Fixing default values in design cycle.

Module 3: Assembly process for HDI-PCBs[2+2 hrs] Through hole and Surface mounting. Attachment methods - Soldering - wave and IR methods. Wire bonding. TAB attach. Flip-chip attach. Substrate behaviour. Coplanarity issues. Cleaning of assembled boards.

Module 4: Thermal behaviour for HDI-PCBs [ $2+2$ hrs] Heat generation by electronic packages. Thermal cycling. Substrate limitations. Thermal failure analysis. Joint reliability estimations. Board cooling methods.

Module 5: Electrical behaviour for HDI-PCBs $[2+2$ hrs] High speeds signal transmission. Considerations of behaviour of wiring board as electrical element. Impedance controlled boards. Appreciation of process latitudes in electrical design.

Module 6: Testing of HDI-PCBs[2 hrs] Description of testing methods. Bed-of nails testing. Fixtures for testing. Flying probe testing. Generation of test networks.

Module 7: Integrated approach for HDI-PCBs [2 hrs] Overall concepts of DFM. Applying DFM guidelines.

Module 8: Design documentation [2 $\mathrm{hrs}]$ Understanding fabricator language. Board specifications. Cost implication of design decisions. Documentation, standards. Generation of CAD data for manufacturing.

Laboratory Exercises: Designing for manufacturability is essentially a laboratory practice. It is difficult to create manufacturing environment in colleges and institutions where students are made to learn engineering design.

\section{Conclusions}

Design for manufacturability of electronic products is highly inter-disciplinary. It is not possible to offer a formal course on such a topic because of difficulties in getting the required multi-faceted expertise, and also to create factorylike situations in formal institutional learning atmosphere.
Therefore, there is a strong case for the proposed web-based course on DFM, which can be offered as a second level course for those interested engineers in electronics packaging.

\section{References:}

1. A course on Introduction to Electronics Packaging, N.J.Rao, and G.Ananda Rao, ETTC conference, 1998.

2. Designers drive DFM, Nick Lester, Circuits Assenably Asia, Nov-Dec-1996

3. The Evolution of New Design Standards, Gary Ferrrai, Circuits Assembly Asia, June 1997.

4. Design for manufacturability, Ray Prasad, Surface Mount Components, Aug-1996

\section{COLOR}

The CD-ROM version of this paper contains color, to assist you in interpretation. http://www.cpmt.org/proceedings/order.html 